\title{
Prediction of Small-For-Gestational-Age Neonates at 33-39 Weeks' Gestation in China: Logistic Regression Modeling of The Contributions of Second- and Third-Trimester Ultrasound Data and Maternal Factors
}

\author{
Danping Xu ( 411087356@qq.com) \\ Taizhou Hospital of Zhejiang Province, Wezhou Medical University \\ Xiuzhen Shen \\ Taizhou Hospital of Zhejiang Province, Wezhou Medical University \\ Heqin Guan \\ Taizhou Hospital of Zhejiang Province, Wezhou Medical University \\ Yiyang Zhu \\ Taizhou Hospital of Zhejiang Province, Wezhou Medical University \\ Minchan Yan \\ Taizhou Hospital of Zhejiang Province, Wezhou Medical University \\ Xiafang Wu \\ Taizhou Hospital of Zhejiang Province, Wezhou Medical University
}

\section{Research Article}

Keywords: small-for-gestational-age, ultrasound, estimated fetal weight, abdomen circumference, third-trimester screening

Posted Date: January 31st, 2022

DOI: https://doi.org/10.21203/rs.3.rs-1158150/v1

License: (c) (i) This work is licensed under a Creative Commons Attribution 4.0 International License. Read Full License

Version of Record: A version of this preprint was published at BMC Pregnancy and Childbirth on August 25th, 2022. See the published version at https://doi.org/10.1186/s12884-022-04991-7. 


\section{Abstract}

Objectives A screening model for prediction of small-for-gestational-age (SGA) neonates (SGAp) was established by logistic regression using ultrasound data and maternal factors (MF). We aimed to evaluate the ability of SGAp as well as abdominal circumference (AC) and estimated fetal weight (EFW) measurements to predict SGA neonates at 33-39 weeks' gestation.

Methods This retrospective study evaluated 5298 singleton pregnancies that had involved three ultrasound examinations at $21^{+0}-27^{+6}, 28^{+0}$ $32^{+6}$, and $33^{+0}-39^{+6}$ weeks. All ultrasound data were transformed to MoM values (multiple of the median). Multivariate logistic regression was used to analyze the correlation between SGA status and various variables (ultrasound data and MF) during pregnancy to build the SGAp model. EFW was calculated according to the Hadlock formula at 33-39 weeks of gestation. The predictive performance of SGAp, AC MoM value at $33^{+0}-39^{+6}$ weeks (AC-M), EFW MoM value (EFW-M), and EFW-M plus MF was evaluated using ROC curves, the detection rates (DRs) at false-positive rates (FPRs) of $5 \%$ and $10 \%$, and the FPRs at DRs of $85 \%, 90 \%$, and $95 \%$.

Results The AUCs of SGAp, AC-M, EFW-M, and EFW-M plus MF for SGA neonates screening were 0.933 (95\%Cl: 0.916-0.950), 0.906 (95\%Cl: 0.887-0.925), 0.920 (95\%Cl: 0.903-0.936), and 0.925 (95\%Cl: 0.909-0.941), respectively. The DRs of SGAp, AC-M, EFW-M, and EFW-M plus MF for SGA neonates were $80.4 \%, 69.6 \%, 73.8 \%$ and $74.3 \%$ at $10 \%$ FPR. The AUCs of SGAp, AC-M, EFW-M, and EFW-M plus MF 0.950 (95\%Cl: 0.932-0.967), 0.929 (95\% Cl: 0.909-0.949), 0.938 (95\%Cl: 0.921-0.956) and 0.941 (95\%Cl: 0.924-0.958), respectively for screening SGA neonates delivered within 2 weeks after the assessment. The DRs for these births increased to $85.8 \%, 75.8 \%, 80.0 \%$, and $82.5 \%$, respectively.

Conclusion The rational use of ultrasound data can significantly improve the prediction of SGA statuses.

\section{Introduction}

Small-for-gestational-age (SGA) status is defined by birth weight below the 10th percentile of the mean weight for the same gestational age (GA) or two standard deviations below the mean weight for the same GA. It is one of the most common clinical neonatal diseases. The mean incidence of the SGA status among infants is approximately $10 \%$, and the SGA status is associated with more risk factors and more complications. SGA is a crucial predictive index of surgical intervention for congenital heart disease (CHD), and the coexistence of SGA and CHD is more likely to lead to death than either alone [1, 2]. One study published in 1997 reported that the incidence of suspected fetal asphyxia was 3 -fold $(6 \%-8 \%)$ higher in SGA fetuses than in non-SGA fetuses [3]. The perinatal mortality rate of SGA fetuses was high, and survivors showed adverse neurocognitive development leading to non-severe neurological dysfunction [4,5]. Several approaches have been used to predict SGA neonates during pregnancy, some of which are summarized below.

1. Estimated fetal weight (EFW) measurements: The fetal weight can be calculated using different combinations of the sonographic values of head circumference (HC), abdominal circumference (AC), femur length (FL), and biparietal diameter (BPD) during pregnancy [6-8]. Most EFW formulas show a strong correlation between the predicted weight and actual birth weight ( $r>0.9,19 / 21$ formulas) [6]. The area under the curve (AUC) for predictions based on EFW measurements in the mid-trimester or third-trimester ultrasound was $0.69 \sim 0.72$ and 0.79 , respectively $[9,10]$. The combination of an EFW model in the second and third trimesters along with assessment of maternal factors (MF) and placental flow measurements can improve the screening results for SGA (AUC, $0.84 ; 95 \% \mathrm{Cl} 0.82-0.87$ ) [10]. Ciobanu et al. showed that the AUC of EFW measurements was 0.883 at 35 and 36 weeks of gestation, while the AUC for births that occurred within two weeks of the evaluation could be as high as 0.933 [11].

2. Ultrasound data: HC, AC, FL, BPD, AC growth velocity, cerebroplacental ratio (CPR), umbilical arteryc Doppler (UAD), etc. can also be used individually or in different combinations to predict SGA status. The AUC of AC measurements in the mid-trimester ultrasound was only 0.68 [12]. The AUC of the AC growth velocity between 20 and 36 weeks was 0.808 , while the AUC for births within two weeks of the evaluation was 0.884 [11]. The AUCs of UAD or CPR measurements for predicting SGA status were 0.54 and 0.51 [13]. Thus, CPR and UAD assessments showed poor performance for predicting neonatal SGA $[13,14]$.

3. Biomarker evaluations: In early pregnancy, the AUCs of visfatin, soluble fms-like tyrosine kinase 1 (SFLT-1)/placental growth factor (PIGF) ratio, and PIGF for screening SGA neonates were $0.71,0.55$, and 0.65 , respectively, which were not ideal [15]. In the second trimester, the AUCs of alpha-fetoprotein (AFP), beta-human chorionic gonadotropin ( $\beta$-hCG), unconjugated estriol (UE3), and inhibin A (IHA) for predicting SGA neonates $<10$ th percentile were $0.724,0.655,0.597$, and 0.664 , respectively [16]. However, the detection rate (DR) and false positive rate (FPR) for SGA neonates were not good ${ }^{16}$. In late pregnancy (30-34 weeks), the AUCs of PIGF, SFLT-1, $\beta$-hCG, and AFP for SGA neonatal screening were $0.838,0.763,0.692$, and 0.725 , respectively [17]. Thus, PIGF and EFW in conjunction with MF show good predictive value [17].

Nevertheless, considering the limitations of the methods described above, this study is, to our knowledge, the first to use MoM valuetransformed ultrasound data obtained in the second and third trimesters of pregnancy along with maternal history of disease during 
pregnancy to establish an SGA screening model (SGAp) through multivariate logistic regression modeling and thereby improve the accuracy of SGA screening.

\section{Methods}

\section{Study design overview}

This was a cohort study of 5298 singleton pregnancies at Taizhou Hospital between January 2017 and March 2021 . Figure 1 outlines the data collection process. The inclusion criteria were single live births at 33 to 41 weeks' gestation, and a history of at least three ultrasonographic examinations at $21^{+0}-27^{+6}, 28^{+0}-32^{+6}$, and $33^{+0}-39^{+6}$ weeks at our hospital. Maternal characteristics, diseases during the pregnancy, ultrasound data, and delivery information were recorded when the pregnant women came to our hospital for delivery.

The GA of the fetus was determined by the last menstrual period and the crown-to-rump length (CRL) of the fetuses at 11-14 weeks [18]. When the GAs determined by both methods were less than 1 week apart, the last menstrual period was chosen to determine the GA. However, when the GAs determined by both methods were more than 1 week apart, the GA based on ultrasound measurements was included in the analyses. All ultrasound examinations were performed by examiners with a certificate from a medical practitioner. In China, pregnant women younger than 35 weeks were required to participate in the first- and second-trimester screening or NIPT screening, and older and high-risk pregnant women were required to undergo amniocentesis to eliminate major fetal chromosome diseases. All participants signed informed consent and the study was approved by the Institutional Review Board of the Taizhou Hospital of Zhejiang Province in accordance with the Declaration of Helsinki (approval number K20181112).

\section{Maternal characteristics}

All pregnant women were Chinese. Maternal age, height, weight, pregnancy history (the number of pregnancy, number of production, scarred uterus), and disease history during pregnancy (hypertension, diabetes, preeclampsia, intrahepatic cholestasis, chronic viral hepatitis B, pelvic adhesionism, anemia, vaginal inflammation, placental dysfunction) were recorded. The delivery process was also well-documented, and the mode of delivery, fetal distress, vaginal laceration, and the use of low forceps was recorded.

\section{Neonatal characteristics}

Neonatal sex, weight, GA at birth, and neonatal asphyxia were recorded. According to the latest Chinese standards [19], a fetus weighing less than the 10th percentile or more than 90th percentile of the GA ( $\leq 36$ weeks), and weighing less than $2500 \mathrm{~g}$ or more than $4000 \mathrm{~g}$ after term is considered a SGA neonate or large for GA (LGA) neonate, respectively. Neonates that do not meet the criteria for SGA and LGA statuses are called appropriate for gestational age (AGA) neonates.

\section{Transformed ultrasound data according to GA}

All ultrasound data in this paper were transformed according to the local median of gestational ages, that was to say MoM values (multiple of the median). The reasons were as follows: At each GA, ultrasound data were not normally distributed. And each region had distinct differences due to climate and diet differences (e.g., northern and southern China). Therefore, MoM values were more appropriate than Z-Score when ultrasound data were transformed according to GA in this region.

\section{Estimated fetal weight (EFW)}

The combination of AC, FL, BPD and $\mathrm{HC}$ yields a variety of formulas for calculating EFW [20]. According to the ultrasonic data from $33^{+0}-39^{+6}$ weeks' gestation, EFW was calculated respectively and finally converted into EFW MoM values (EFW-M) according to GA. ROC curve analysis showed that the formula created by Hadlock (1985) based on AC, FL and HC was the most suitable for this paper (Data not displayed).

\section{Statistical analysis}

Data were described in terms of medians/interquartile range (IQR) for continuous variables or numbers (n and \%) for categorical variable. Mann-whitney $U$ test, rank sum test or Chi-square test were used to compare differences between groups. Multivariate logistic regression was used to establish a SGA predictor (SGAp) model. Receiver operating characteristic (ROC) curves analysis was performed to evaluate the power of SGAp, AC MoM value at $33^{+0}-39^{+6}$ weeks (AC-M), EFW-M, and EFW-M plus MF to discriminate SGA neonates. A p < 0.05 was considered to be significant.

\section{Results}




\section{Patient characteristics}

A total of 5298 pregnancies with GAs of 33-41 weeks at birth were included in the study (Figure 1 shows the inclusion process). The study population included 214 SGA (4.1\%), 4828 AGA (91.1\%), and 256 LGA (4.8\%) neonates. The AGA and LGA neonates, collectively referred to as non-SGA neonates, served as the control group in this study. Basic information on the pregnant women, disease history during pregnancy, delivery, and newborn information are shown in Table 1. Weight, height, and body mass index (BMI) during pregnancy, proportion of boys, gestational week of delivery, and the proportion of streptococcal vaginitis in the women who delivered SGA neonates were significantly lower than those in women who delivered AGA and LGA neonates. On the other hand, the proportions of cesarean deliveries and patients with chronic hypertension and preeclampsia among women who delivered SGA neonates were significantly higher than those among women who delivered AGA and LGA neonates. Correlation analysis showed that SGA was correlated with maternal height, weight, BMI, fetal sex, and gestational disease history (streptococcus lactis vaginitis, gestational hypertension, preeclampsia, intrahepatic cholestasis during pregnancy), while LGA was associated with maternal age, height, weight, BMI, number of pregnancies, number of deliveries, baby sex, and gestational disease history (gestational diabetes, bacterial vaginitis, anemia). 
Table 1

The characteristics of pregnant women, their pregnancies and neonates.

\begin{tabular}{|c|c|c|c|c|}
\hline \multirow[t]{2}{*}{ Characteristics } & SGA & AGA & LGA & \multirow[t]{2}{*}{$\mathbf{P}$} \\
\hline & $(n=214)$ & $(n=4828)$ & $(n=256)$ & \\
\hline \multicolumn{5}{|l|}{ Maternal characteristics } \\
\hline Age (years) & $29(26-33)$ & $30(26-33)$ & $31(28-34)^{\star \star}$ & $<0.001$ \\
\hline Predelivery weight (kg) & $64(59-72)^{\star \star}$ & $68(63-74)$ & $74(69-80)^{\star \star}$ & $<0.001$ \\
\hline Height (cm) & $158(155-160)^{\star \star}$ & $160(157-163)$ & $160(158-164)^{\star \star}$ & $<0.001$ \\
\hline BMI & $0.4(0.4-0.4)^{\star *}$ & $0.4(0.4-0.5)$ & $0.5(0.4-0.5)^{\star \star}$ & $<0.001$ \\
\hline The number of pregnancy & $2(1-3)$ & $2(1-3)$ & $2(1-4)^{\star \star}$ & 0.014 \\
\hline The number of delivery & $0(0-1)$ & $0(0-1)$ & $1(0-1)^{*}$ & 0.004 \\
\hline \multicolumn{5}{|l|}{ Newborn information } \\
\hline \multicolumn{5}{|l|}{ Baby gender } \\
\hline Boy & $85(39.7 \%)^{\star \star}$ & $2520(52.2 \%)$ & $183(71.5 \%)^{\star \star}$ & \multirow[t]{2}{*}{$<0.001$} \\
\hline Girl & $129(60.3 \%)$ & $2308(47.8 \%)$ & $73(28.5 \%)$ & \\
\hline Gestational age at birth (weeks) & $37(37-38)^{\star \star}$ & $38(37-39)$ & $39(39-40)^{\star *}$ & $<0.001$ \\
\hline Premature infant (33-36 weeks) & $45(21.0 \%)^{\star \star}$ & $418(8.7 \%)$ & $1(0.4 \%)^{\star \star}$ & $<0.001$ \\
\hline The birth weight of the baby & $2355(2195-2440)^{\star \star}$ & $3250(2990-3510)$ & $4160(4070-4300)^{\star \star}$ & $<0.001$ \\
\hline Neonatal asphyxia & $0(0 \%)$ & $15(0.3 \%)$ & $1(0.4 \%)$ & 0.921 \\
\hline \multicolumn{5}{|l|}{ The delivery information } \\
\hline Scarred uterus & $27(12.6 \%)$ & $756(15.7 \%)$ & $47(18.4 \%)$ & 0.233 \\
\hline Caesarean section & $90(42.1 \%)^{\star \star}$ & $1362(28.2 \%)$ & $95(37.1 \%)^{\star \star}$ & $<0.001$ \\
\hline Colpoperineal laceration & $71(33.1 \%)^{\star \star}$ & $2449(50.8 \%)$ & $102(39.9 \%)^{\star \star}$ & $<0.001$ \\
\hline Grade I vaginal laceration & $60(28.0 \%)^{*}$ & $1760(36.5 \%)$ & $58(22.7 \%)^{\star \star}$ & $<0.001$ \\
\hline Grade II vaginal laceration & $11(5.1 \%)^{\star \star}$ & $689(14.3 \%)$ & $44(17.2 \%)$ & $<0.001$ \\
\hline Fetal distress in uterus & $29(13.6 \%)$ & $554(11.5 \%)$ & $26(10.2 \%)$ & 0.511 \\
\hline Low forceps delivery & $5(2.3 \%)$ & $249(5.2 \%)$ & $18(7.0 \%)$ & 0.069 \\
\hline \multicolumn{5}{|l|}{ Diseases of pregnancy } \\
\hline Coleitis & $25(11.7 \%)$ & $559(11.6 \%)$ & $36(14.1 \%)$ & 0.484 \\
\hline Bacterial vaginosis & $6(2.8 \%)$ & $111(2.3 \%)$ & $15(5.9 \%)^{\star \star}$ & $<0.001$ \\
\hline Candida vaginosis & $20(9.3 \%)$ & $310(6.4 \%)$ & $18(7.0 \%)$ & 0.229 \\
\hline Streptococcus lactis vaginitis & $2(0.9 \%)^{*}$ & $201(4.2 \%)$ & $14(5.5 \%)$ & 0.035 \\
\hline Trichomonas vaginitis & $1(0.5 \%)$ & $6(0.1 \%)$ & $1(0.4 \%)$ & 0.269 \\
\hline Gestational diabetes mellitus & $46(21.5 \%)$ & $1088(22.5 \%)$ & $86(33.6 \%)^{\star \star}$ & $<0.001$ \\
\hline Hypertension & $19(8.9 \%)^{\star \star}$ & $189(3.9 \%)$ & $8(3.1 \%)$ & $<0.001$ \\
\hline Pregnancy hypertension & $12(5.6 \%)$ & $168(3.5 \%)$ & $6(2.3 \%)$ & 0.148 \\
\hline Chronic hypertension & $7(3.3 \%)^{\star \star}$ & $21(0.4 \%)$ & $2(0.8 \%)$ & $<0.001$ \\
\hline
\end{tabular}




\begin{tabular}{|c|c|c|c|c|}
\hline \multirow[t]{2}{*}{ Characteristics } & SGA & AGA & LGA & \multirow[t]{2}{*}{$P$} \\
\hline & $(n=214)$ & $(n=4828)$ & $(n=256)$ & \\
\hline Hypoplacental function & $30(14.0 \%)$ & $554(11.5 \%)$ & $22(8.6 \%)$ & 0.177 \\
\hline Preeclampsia & $29(13.6 \%)^{* \star}$ & $118(2.4 \%)$ & $7(2.7 \%)$ & $<0.001$ \\
\hline Mild preeclampsia & $12(5.6 \%)^{\star \star}$ & $81(1.7 \%)$ & $7(2.7 \%)$ & $<0.001$ \\
\hline Serious preeclampsia & $17(7.9 \%)^{\star \star}$ & $37(0.8 \%)$ & $0(0 \%)$ & $<0.001$ \\
\hline Intrahepatic cholestasis during pregnancy & $8(3.7 \%)$ & $79(1.6 \%)$ & $2(0.8 \%)$ & 0.076 \\
\hline Thyroid dysfunction & $11(5.1 \%)$ & $411(8.5 \%)$ & $23(9 \%)$ & 0.368 \\
\hline \multicolumn{5}{|l|}{ Virus infection } \\
\hline HBV & $5(2.3 \%)$ & $111(2.3 \%)$ & $6(2.3 \%)$ & 0.998 \\
\hline Syphilis & $1(0.5 \%)$ & $26(0.5 \%)$ & $1(0.4 \%)$ & 0.943 \\
\hline HPV & $0(0 \%)$ & $12(0.2 \%)$ & $1(0.4 \%)$ & 0.688 \\
\hline Abnormal shape of placenta & $19(8.9 \%)$ & $343(7.1 \%)$ & $18(7.0 \%)$ & 0.678 \\
\hline Pelvic adhesion & $3(1.4 \%)$ & $97(2.0 \%)$ & $6(2.3 \%)$ & 0.761 \\
\hline Anemia & $18(8.4 \%)$ & $562(11.6 \%)$ & $40(15.6 \%)$ & 0.048 \\
\hline
\end{tabular}

\section{Ultrasound data at different gestational weeks}

All pregnant women had undergone ultrasound examinations at $20^{+0}-27^{+6}$ weeks, $28^{+0}-32^{+6}$ weeks, and $33^{+0}-39^{+6}$ weeks. In China, the most accurate detection time for abnormal ultrasound findings is $23-25$ weeks, so a large number of people choose to undergo ultrasound at 24 weeks' gestation. All data were grouped according to GA and described as median, 10th, and 90th percentiles (Table 2). After all data were MoM value-transformed, rank-sum test analysis showed that the BPD, OFD, HC, FL, AC, and amniotic fluid index (AFI) of SGA fetuses were significantly lower than those of AGA and LGA fetuses at $20^{+0}-27^{+6}, 28^{+0}-32^{+6}$, and $33^{+0}-39^{+6}$ weeks' gestation (Table 3 ). 
Table 2

the 10th percentile, 50th percentile and 90th percentile values of $\mathrm{BPD}, \mathrm{OFD}, \mathrm{HC}, \mathrm{FL}, \mathrm{AC}, \mathrm{AFI}, \mathrm{S} / \mathrm{D}, \mathrm{PI}, \mathrm{RI}$ and $\mathrm{PT}$ for gestational age.

\begin{tabular}{|c|c|c|c|c|c|c|c|c|c|c|c|c|c|c|c|c|}
\hline \multirow[t]{2}{*}{ GA } & \multirow[t]{2}{*}{$\mathbf{N}$} & \multicolumn{3}{|l|}{ BPD } & \multicolumn{3}{|l|}{ OFD } & \multicolumn{3}{|l|}{$\mathrm{HC}$} & \multicolumn{3}{|l|}{$\mathrm{FL}$} & \multicolumn{3}{|l|}{$A C$} \\
\hline & & 10th & 50 th & 90th & 10th & 50 th & 90th & 10th & 50th & 90th & 10 th & 50th & 90th & 10 th & 50th & 90th \\
\hline $21^{+0} \sim 21^{+6}$ & 11 & 48 & 52 & 55 & 62 & 66 & 69 & 177 & 190 & 205 & 33 & 37 & 38 & 158 & 167 & 179 \\
\hline $22^{+0} \sim 22^{+6}$ & 56 & 53 & 55 & 59 & 67 & 71 & 75 & 196 & 207 & 218 & 37 & 40 & 42 & 171 & 183 & 193 \\
\hline $23^{+0} \sim 23^{+6}$ & 887 & 54 & 59 & 63 & 70 & 74 & 79 & 205 & 218 & 230 & 39 & 42 & 45 & 178 & 192 & 206 \\
\hline $24^{+0} \sim 24^{+6}$ & 3549 & 57 & 60 & 64 & 72 & 76 & 80 & 213 & 223 & 233 & 41 & 43 & 46 & 187 & 198 & 209 \\
\hline $25^{+0} \sim 25^{+6}$ & 684 & 59 & 62 & 66 & 75 & 79 & 83 & 221 & 231 & 242 & 43 & 45 & 48 & 194 & 206 & 217 \\
\hline $26^{+0} \sim 26^{+6}$ & 80 & 61 & 65 & 68 & 77 & 82 & 86 & 229 & 240 & 252 & 44 & 47 & 50 & 197 & 214 & 224 \\
\hline $27^{+0} \sim 27^{+6}$ & 31 & 58 & 68 & 73 & 74 & 87 & 91 & 218 & 254 & 264 & 42 & 50 & 52 & 195 & 226 & 240 \\
\hline $28^{+0} \sim 28^{+6}$ & 686 & 69 & 72 & 76 & 87 & 91 & 95 & 255 & 265 & 277 & 51 & 53 & 56 & 230 & 242 & 255 \\
\hline $29^{+0} \sim 29^{+6}$ & 1200 & 72 & 75 & 79 & 90 & 94 & 98 & 264 & 275 & 287 & 53 & 55 & 58 & 241 & 253 & 267 \\
\hline $30^{+0} \sim 30^{+6}$ & 1708 & 74 & 77 & 81 & 92 & 96 & 101 & 272 & 282 & 293 & 55 & 57 & 60 & 250 & 262 & 275 \\
\hline $31^{+0} \sim 31^{+6}$ & 915 & 76 & 80 & 83 & 95 & 99 & 104 & 279 & 289 & 301 & 57 & 59 & 62 & 259 & 273 & 285 \\
\hline $32^{+0} \sim 32^{+6}$ & 789 & 78 & 82 & 86 & 97 & 102 & 106 & 286 & 297 & 309 & 59 & 61 & 63 & 267 & 282 & 295 \\
\hline $33^{+0} \sim 33^{+6}$ & 589 & 80 & 84 & 88 & 100 & 104 & 109 & 292 & 304 & 317 & 60 & 63 & 66 & 278 & 293 & 307 \\
\hline $34^{+0} \sim 34^{+6}$ & 1333 & 82 & 86 & 90 & 102 & 106 & 110 & 300 & 310 & 321 & 63 & 65 & 68 & 288 & 302 & 317 \\
\hline $35^{+0} \sim 35^{+6}$ & 1386 & 85 & 88 & 92 & 104 & 108 & 112 & 306 & 316 & 328 & 64 & 67 & 69 & 297 & 312 & 327 \\
\hline $36^{+0} \sim 36^{+6}$ & 1165 & 86 & 90 & 93 & 106 & 110 & 113 & 311 & 321 & 333 & 66 & 69 & 71 & 306 & 320 & 335 \\
\hline $37^{+0} \sim 37^{+6}$ & 574 & 88 & 92 & 95 & 108 & 112 & 115 & 316 & 327 & 338 & 67 & 70 & 72 & 314 & 330 & 345 \\
\hline $38^{+0} \sim 38^{+6}$ & 206 & 88 & 92 & 96 & 108 & 112 & 116 & 318 & 329 & 342 & 68 & 71 & 74 & 320 & 334 & 351 \\
\hline $39^{+0} \sim 39^{+6}$ & 45 & 90 & 94 & 98 & 109 & 114 & 118 & 322 & 336 & 347 & 69 & 72 & 74 & 327 & 342 & 358 \\
\hline \multirow[t]{2}{*}{ GA } & \multirow[t]{2}{*}{$\mathrm{N}$} & \multicolumn{3}{|l|}{ AFI } & \multicolumn{3}{|l|}{$S / D$} & \multicolumn{3}{|l|}{ PI } & \multicolumn{3}{|l|}{ RI } & \multicolumn{3}{|l|}{ PT } \\
\hline & & 10th & 50 th & 90th & 10th & 50th & 90th & 10th & 50th & 90th & 10th & 50th & 90th & 10th & 50th & 90th \\
\hline $21^{+0} \sim 21^{+6}$ & 11 & 95 & 116 & 127 & 2.95 & 3.11 & 3.80 & 1.03 & 1.11 & 1.29 & 0.66 & 0.68 & 0.74 & 21 & 22 & 25 \\
\hline $22^{+0} \sim 22^{+6}$ & 56 & 94 & 119 & 141 & 2.46 & 2.96 & 3.59 & 0.90 & 1.08 & 1.26 & 0.60 & 0.67 & 0.72 & 21 & 24 & 28 \\
\hline $23^{+0} \sim 23^{+6}$ & 887 & 92 & 119 & 157 & 2.38 & 3.05 & 3.84 & 0.85 & 1.08 & 1.28 & 0.58 & 0.67 & 0.74 & 20 & 25 & 31 \\
\hline $24^{+0} \sim 24^{+6}$ & 3549 & 96 & 119 & 147 & 2.46 & 3.00 & 3.65 & 0.88 & 1.06 & 1.24 & 0.59 & 0.67 & 0.73 & 22 & 26 & 30 \\
\hline $25^{+0} \sim 25^{+6}$ & 684 & 96 & 119 & 148 & 2.46 & 2.96 & 3.57 & 0.88 & 1.05 & 1.22 & 0.59 & 0.66 & 0.72 & 22 & 26 & 31 \\
\hline $26^{+0} \sim 26^{+6}$ & 80 & 95 & 117 & 149 & 2.3 & 2.84 & 3.50 & 0.81 & 1.02 & 1.18 & 0.57 & 0.65 & 0.71 & 23 & 27 & 32 \\
\hline $27^{+0} \sim 27^{+6}$ & 31 & 96 & 110 & 134 & 2.38 & 2.69 & 3.30 & 0.86 & 0.99 & 1.14 & 0.58 & 0.63 & 0.70 & 24 & 28 & 31 \\
\hline $28^{+0} \sim 28^{+6}$ & 686 & 94 & 115 & 144 & 2.18 & 2.68 & 3.24 & 0.77 & 0.95 & 1.14 & 0.54 & 0.63 & 0.69 & 25 & 29 & 33 \\
\hline $29^{+0} \sim 29^{+6}$ & 1200 & 92 & 112 & 143 & 2.14 & 2.62 & 3.10 & 0.75 & 0.94 & 1.09 & 0.53 & 0.62 & 0.68 & 26 & 30 & 34 \\
\hline $30^{+0} \sim 30^{+6}$ & 1708 & 90 & 110 & 141 & 2.09 & 2.53 & 2.95 & 0.73 & 0.91 & 1.06 & 0.52 & 0.61 & 0.66 & 27 & 31 & 35 \\
\hline
\end{tabular}




\begin{tabular}{|c|c|c|c|c|c|c|c|c|c|c|c|c|c|c|c|c|}
\hline \multirow[t]{2}{*}{ GA } & \multirow[t]{2}{*}{$\mathbf{N}$} & \multicolumn{3}{|l|}{ BPD } & \multicolumn{3}{|l|}{ OFD } & \multicolumn{3}{|l|}{$\mathrm{HC}$} & \multicolumn{3}{|l|}{$\mathrm{FL}$} & \multicolumn{3}{|l|}{$A C$} \\
\hline & & 10th & 50th & 90th & 10th & 50th & 90th & 10th & 50th & 90th & 10th & 50th & 90th & 10th & 50th & 90th \\
\hline $31^{+0} \sim 31^{+6}$ & 915 & 87 & 108 & 143 & 2.05 & 2.49 & 2.93 & 0.71 & 0.90 & 1.05 & 0.51 & 0.60 & 0.66 & 27 & 31 & 36 \\
\hline $32^{+0} \sim 32^{+6}$ & 789 & 85 & 105 & 136 & 2.01 & 2.46 & 2.88 & 0.69 & 0.89 & 1.03 & 0.50 & 0.59 & 0.65 & 28 & 32 & 37 \\
\hline $33^{+0} \sim 33^{+6}$ & 589 & 78 & 102 & 136 & 1.97 & 2.38 & 2.84 & 0.68 & 0.86 & 1.02 & 0.49 & 0.58 & 0.65 & 29 & 33 & 38 \\
\hline $34^{+0} \sim 34^{+6}$ & 1333 & 80 & 101 & 134 & 1.92 & 2.32 & 2.79 & 0.65 & 0.84 & 1.02 & 0.48 & 0.57 & 0.64 & 29 & 33 & 38 \\
\hline $35^{+0} \sim 35^{+6}$ & 1386 & 77 & 100 & 130 & 1.91 & 2.26 & 2.75 & 0.64 & 0.81 & 1.00 & 0.48 & 0.56 & 0.64 & 30 & 34 & 38 \\
\hline $36^{+0} \sim 36^{+6}$ & 1165 & 78 & 99 & 130 & 1.88 & 2.24 & 2.73 & 0.63 & 0.81 & 0.99 & 0.47 & 0.55 & 0.63 & 30 & 34 & 39 \\
\hline $37^{+0} \sim 37^{+6}$ & 574 & 76 & 98 & 135 & 1.82 & 2.18 & 2.66 & 0.60 & 0.78 & 0.97 & 0.45 & 0.54 & 0.62 & 31 & 35 & 39 \\
\hline $38^{+0} \sim 38^{+6}$ & 206 & 71 & 99 & 135 & 1.84 & 2.16 & 2.61 & 0.61 & 0.78 & 0.98 & 0.46 & 0.54 & 0.62 & 31 & 35 & 38 \\
\hline $39^{+0} \sim 39^{+6}$ & 45 & 63 & 90 & 125 & 1.82 & 2.11 & 2.52 & 0.61 & 0.77 & 0.96 & 0.45 & 0.53 & 0.61 & 31 & 35 & 39 \\
\hline
\end{tabular}


Table 3

The BPD, OFD, HC, FL, AC, AFI, S/D, PI, RI MoM values were converted according to gestational age.

\begin{tabular}{|c|c|c|c|c|c|}
\hline \multirow[t]{2}{*}{ GA } & \multirow[t]{2}{*}{ Parameters } & \multirow{2}{*}{$\begin{array}{l}S G A \\
(n=214)\end{array}$} & \multirow{2}{*}{$\begin{array}{l}\text { AGA } \\
(n=4828)\end{array}$} & \multirow{2}{*}{$\begin{array}{l}\text { LGA } \\
(n=256)\end{array}$} & \multirow[t]{2}{*}{$P$} \\
\hline & & & & & \\
\hline \multirow[t]{10}{*}{$21^{+0} \sim 27^{+6}$} & BPD & $0.98(0.95-1.00)^{\star *}$ & $1.00(0.97-1.03)$ & $1.02(0.98-1.05)^{\star \star}$ & $<0.001$ \\
\hline & OFD & $0.97(0.94-1.00)^{* k}$ & $1.00(0.97-1.03)$ & $1.01(0.99-1.04)^{\star \star}$ & $<0.001$ \\
\hline & $\mathrm{HC}$ & $0.98(0.95-1.00)^{* *}$ & $1.00(0.98-1.02)$ & $1.02(0.99-1.04)^{\star *}$ & $<0.001$ \\
\hline & $\mathrm{FL}$ & $0.98(0.95-1.00)^{* *}$ & $1.00(0.98-1.04)$ & $1.02(1.00-1.05)^{\star *}$ & $<0.001$ \\
\hline & $A C$ & $0.98(0.94-1.00)^{\star *}$ & $1.00(0.97-1.03)$ & $1.03(1.00-1.06)^{\star *}$ & $<0.001$ \\
\hline & AFI & $0.95(0.84-1.06)^{* k}$ & $1.00(0.89-1.12)$ & $1.03(0.94-1.14)^{\star \star}$ & $<0.001$ \\
\hline & $S / D$ & $1.01(0.89-1.16)$ & $1.00(0.90-1.11)$ & $0.97(0.86-1.04)^{\star *}$ & $<0.001$ \\
\hline & $\mathrm{PI}$ & $1.00(0.90-1.11)$ & $1.00(0.91-1.09)$ & $0.98(0.88-1.05)^{\star \star}$ & $<0.001$ \\
\hline & RI & $1.00(0.94-1.07)$ & $1.00(0.94-1.04)$ & $0.99(0.91-1.02)^{\star *}$ & $<0.001$ \\
\hline & PT & $0.96(0.88-1.04)^{* *}$ & $1.00(0.92-1.08)$ & $1.04(0.96-1.12)^{\star \star}$ & $<0.001$ \\
\hline \multirow[t]{10}{*}{$28^{+0} \sim 32^{+6}$} & BPD & $0.96(0.94-0.99)^{* k}$ & $1.00(0.98-1.03)$ & $1.01(1.00-1.05)^{\star *}$ & $<0.001$ \\
\hline & OFD & $0.97(0.94-0.99)^{\star *}$ & $1.00(0.98-1.02)$ & $1.02(0.99-1.04)^{\star \star}$ & $<0.001$ \\
\hline & $\mathrm{HC}$ & $0.96(0.95-0.99)^{* *}$ & $1.00(0.98-1.02)$ & $1.02(1.00-1.04)^{\star *}$ & $<0.001$ \\
\hline & $\mathrm{FL}$ & $0.97(0.95-1.00)^{* *}$ & $1.00(0.98-1.03)$ & $1.02(1.00-1.05)^{\star \star}$ & $<0.001$ \\
\hline & $\mathrm{AC}$ & $0.95(0.93-0.98)^{* *}$ & $1.00(0.98-1.02)$ & $1.04(1.01-1.06)^{\star \star}$ & $<0.001$ \\
\hline & AFI & $0.98(0.82-1.12)^{\star}$ & $1.00(0.89-1.13)$ & $1.07(0.97-1.23)^{\star *}$ & $<0.001$ \\
\hline & $S / D$ & $1.05(0.96-1.14)^{* *}$ & $1.00(0.90-1.09)$ & $0.96(0.87-1.06)^{\star *}$ & $<0.001$ \\
\hline & $\mathrm{PI}$ & $1.05(0.96-1.14)^{\star \star}$ & $1.00(0.89-1.09)$ & $0.96(0.86-1.05)^{\star *}$ & $<0.001$ \\
\hline & RI & $1.03(0.97-1.08)^{\star \star}$ & $1.00(0.93-1.05)$ & $0.97(0.90-1.03)^{\star *}$ & $<0.001$ \\
\hline & PT & $0.97(0.90-1.06)$ & $1.00(0.93-1.06)$ & $1.03(0.97-1.12)^{\star *}$ & $<0.001$ \\
\hline \multirow[t]{10}{*}{$33^{+0} \sim 39^{+6}$} & BPD & $0.95(0.93-0.98)^{* *}$ & $1.00(0.98-1.02)$ & $1.02(1.00-1.05)^{\star \star}$ & $<0.001$ \\
\hline & OFD & $0.96(0.95-0.99)^{* *}$ & $1.00(0.98-1.02)$ & $1.02(1.00-1.04)^{* \star}$ & $<0.001$ \\
\hline & $\mathrm{HC}$ & $0.96(0.94-0.98)^{* *}$ & $1.00(0.98-1.02)$ & $1.02(1.01-1.04)^{* *}$ & $<0.001$ \\
\hline & $\mathrm{FL}$ & $0.96(0.93-0.99)^{* *}$ & $1.00(0.98-1.02)$ & $1.02(1.00-1.03)^{\star *}$ & $<0.001$ \\
\hline & $A C$ & $0.93(0.91-0.96)^{* *}$ & $1.00(0.98-1.02)$ & $1.05(1.03-1.07)^{\star *}$ & $<0.001$ \\
\hline & AFI & $0.88(0.77-1.01)^{\star *}$ & $1.00(0.89-1.15)$ & $1.11(0.97-1.33)^{* *}$ & $<0.001$ \\
\hline & $S / D$ & $1.05(0.96-1.19)^{\star \star}$ & $1.00(0.91-1.11)$ & $0.95(0.86-1.06)^{\star *}$ & $<0.001$ \\
\hline & $\mathrm{PI}$ & $1.05(0.95-1.19)^{\star *}$ & $1.00(0.88-1.12)$ & $0.94(0.82-1.05)^{\star *}$ & $<0.001$ \\
\hline & $\mathrm{RI}$ & $1.04(0.96-1.11)^{\star *}$ & $1.00(0.91-1.07)$ & $0.96(0.87-1.04)^{\star *}$ & $<0.001$ \\
\hline & PT & $0.97(0.91-1.06)^{\star \star}$ & $1.00(0.94-1.06)$ & $1.03(1.00-1.11)^{\star \star}$ & $<0.001$ \\
\hline
\end{tabular}




\begin{tabular}{|c|c|c|c|c|c|}
\hline \multirow[t]{2}{*}{ GA } & \multirow[t]{2}{*}{ Parameters } & SGA & AGA & LGA & \multirow[t]{2}{*}{$P$} \\
\hline & & $(n=214)$ & $(n=4828)$ & $(n=256)$ & \\
\hline
\end{tabular}

\section{Logistic regression modeling}

Logistic regression modeling was conducted for all factors and ultrasound data after transformation. SGA neonates were represented by the dichotomous variable. It was found that the variables significantly correlated with the history of hypertension in pregnant women $\left(X_{1}\right.$, normal blood pressure $=0$, gestational hypertension $=1$, chronic hypertension $=2)$, preeclampsia $\left(X_{2}\right.$, no preeclampsia $=0$, mild disease $=1$, severe disease = 2), OFD MoM value at $21^{+0}-27^{+6}$ weeks (OFD-M-[21, 27]) $\left(X_{3}\right)$, FL MoM value at $21^{+0}-27^{+6}$ weeks (FL-M-[21, 27]) $\left(X_{4}\right)$, AC MoM value at $28^{+0}-32^{+6}$ weeks (AC-M-[28, 32]) $\left(\mathrm{X}_{5}\right)$, BPD MoM value at $33^{+0}-39^{+6}$ weeks (BPD-M) $\left(\mathrm{X}_{6}\right)$, FL MoM value at $33^{+0}-39^{+6}$ weeks (FL-M) $\left(\mathrm{X}_{7}\right)$, AC MoM value at $33^{+0}-39^{+6}$ weeks (AC-M) $\left(\mathrm{X}_{8}\right)$, AFI MoM value at $33^{+0}-39^{+6}$ weeks (AFI-M) $\left(\mathrm{X}_{9}\right)$. This model is called the SGA predictor (SGAp) (As shown in the Table 4). SGAp=-59.496-0.784X $\mathrm{X}_{1}-0.693 \mathrm{X}_{2}-9.377 \mathrm{X}_{3}+7.26 \mathrm{X}_{4}+14.578 \mathrm{X}_{5}+14.903 \mathrm{X}_{6}+8.436 \mathrm{X}_{7}+26.531 \mathrm{X}_{8}+2.087 \mathrm{X}_{9}$.

Table 4

Multivariate Logistic regression model for SGA neonatal prediction.

\begin{tabular}{|c|c|c|c|c|c|}
\hline \multicolumn{2}{|c|}{ Characteristics } & \multirow{2}{*}{$\begin{array}{l}\boldsymbol{\beta} \\
-0.784\end{array}$} & \multirow{2}{*}{$\begin{array}{l}\text { OR } \\
0.46\end{array}$} & \multirow{2}{*}{$\begin{array}{l}95 \% \mathrm{Cl} \\
0.29-0.73\end{array}$} & \multirow{2}{*}{$\begin{array}{l}\mathbf{P} \\
0.001\end{array}$} \\
\hline $\mathrm{X}_{1}$ & Hypertension & & & & \\
\hline$x_{2}$ & Preeclampsia & -0.693 & 0.5 & $0.34-0.74$ & 0.001 \\
\hline$x_{3}$ & OFD-M-[21, 27] & -9.377 & 0 & $0-0.01$ & $<0.001$ \\
\hline $\mathrm{X}_{4}$ & FL-M-[21, 27] & 7.26 & 1422 & $13.11-1.54 * 10^{5}$ & 0.002 \\
\hline$x_{5}$ & AC-M-[28, 32] & 14.578 & $2.14 * 10^{6}$ & $1.11 * 10^{4}-4.16 * 10^{8}$ & $<0.001$ \\
\hline$x_{6}$ & BPD-M & 14.903 & $2.97 * 10^{6}$ & $1.04 * 10^{4}-8.44 * 10^{8}$ & $<0.001$ \\
\hline$x_{7}$ & FL-M & 8.436 & 4609 & $11.24-1.89 * 10^{6}$ & 0.006 \\
\hline$x_{8}$ & AC-M & 26.531 & $3.33 * 10^{11}$ & $6.35^{\star} 10^{8}-1.75^{\star} 10^{14}$ & $<0.001$ \\
\hline$X_{9}$ & AFI-M & 2.087 & 8.06 & $3.44-18.91$ & $<0.001$ \\
\hline \multicolumn{2}{|c|}{ Constant } & -59.496 & 0 & & $<0.001$ \\
\hline \multicolumn{6}{|c|}{$\begin{array}{l}\text { OFD-M-[21, 27], OFD MoM value at } 21^{+0} \sim 27^{+6} \text { weeks; FL-M-[21, 27], FL MoM value at } 21^{+0} \sim 27^{+6} \text { weeks; AC-M-[28, 32], AC MoM value at } \\
28^{+0} \sim 32^{+6} \text { weeks; BPD-M, BPD MoM value at } 33^{+0} \sim 39^{+6} \text { weeks; FL-M, FL MoM value at } 33^{+0} \sim 39^{+6} \text { weeks; AC-M, AC MoM value at } \\
33^{+0} \sim 39^{+6} \text { weeks; AFI-M, AFI MoM value at } 33^{+0} \sim 39^{+6} \text { weeks. }\end{array}$} \\
\hline
\end{tabular}

\section{Prediction of SGA neonate by SGAp}

The SGAp values, AC, AC-M, EFW-M, and EFW-M plus MF of fetuses in the SGA group were significantly lower than those in the non-SGA group (Table 5). The SGAp values were significantly different between the SGA and non-SGA groups (Table 5). The AUCs and the DRs of SGAp, ACM, EFW-M, and EFW-M plus MF for SGA neonate screening at 5\% and 10\% FPR are shown in Table 6 and Figure 2. The corresponding FPRs of these four indicators at $85 \%, 90 \%$, and $95 \%$ DRs are also shown in Table 7. 
Table 5

The median of SGAp, AC-M, EFW-M and EFW-M plus maternal factors in SGA group and non-SGA group.

\begin{tabular}{|lll|}
\hline Characteristic & SGA & non-SGA \\
\cline { 2 - 3 } & median (IQR) & median (IQR) \\
\hline SGA born at any stage $(\mathrm{N})$ & 214 & 5084 \\
\hline SGAp & $0.96(-0.47-2.39)$ & $5.14(3.91-6.36)$ \\
\hline AC-M & $0.93(0.91-0.96)$ & $1.00(0.98-1.03)$ \\
\hline EFW-M & $0.84(0.79-0.90)$ & $1.00(0.95-1.06)$ \\
\hline EFW-M plus MF & $34.56(33.42-36.12)$ & $38.38(37.19-39.56)$ \\
\hline SGA born within 2 weeks $(\mathrm{N})$ & 120 & 1459 \\
\hline SGAp & $0.61(-0.79-1.93)$ & $5.08(3.91-6.35)$ \\
\hline AC-M & $0.93(0.91-0.96)$ & $1.00(0.98-1.03)$ \\
\hline EFW-M & $0.82(0.78-0.89)$ & $1.01(0.95-1.06)$ \\
\hline EFW-M plus MF & $34.35(33.40-35.68)$ & $38.43(37.28-39.62)$ \\
\hline AC-M, AC MoM value at $33^{+0} \sim 39^{+6}$ weeks; EFW-M, EFW MoM value at $33^{+0} \sim 39^{+6}$ weeks; EFW-M plus MF, EFW-M plus maternal factors. \\
\hline
\end{tabular}

Table 6

The predictive performance of SGAp, AC-M, EFW-M and EFW-M plus maternal factors for SGA neonates.

\begin{tabular}{|c|c|c|c|}
\hline screening test & AUC (95\%Cl) & DR at 5\% FPR (\%) & DR at $10 \%$ FPR (\%) \\
\hline \multicolumn{4}{|c|}{ SGA born at any stage } \\
\hline SGAp & $0.933(0.916-0.950)$ & 70.6 (67.4-73.7) & $80.4(77.7-83.1)$ \\
\hline AC-M & $0.906(0.887-0.925)$ & 60.7 (57.4-64.1) & $69.6(66.5-72.8)$ \\
\hline EFW-M & $0.920(0.903-0.936)$ & $66.4(63.1-69.6)$ & 73.8 (70.8-76.8) \\
\hline EFW-M plus MF & $0.925(0.909-0.941)$ & $67.8(64.6-71.0)$ & 74.3 (71.3-77.3) \\
\hline \multicolumn{4}{|c|}{ SGA born within 2 weeks } \\
\hline SGAp & $0.950(0.932-0.967)$ & $74.2(70.2-78.2)$ & 85.8 (82.7-89.0) \\
\hline AC-M & $0.929(0.909-0.949)$ & $66.7(62.4-71.0)$ & 75.8 (71.9-79.7) \\
\hline EFW-M & $0.938(0.921-0.956)$ & 70.8 (66.7-75.0) & $80.0(76.3-83.7)$ \\
\hline EFW-M plus MF & $0.941(0.924-0.958)$ & $72.5(68.4-76.6)$ & $82.5(79.0-86.0)$ \\
\hline
\end{tabular}


Table 7

The false positive rate necessary to achieve prediction of $85 \%, 90 \%$ and $95 \%$ SGA neonates.

\begin{tabular}{|c|c|c|c|}
\hline screening test & FPR for $85 \%$ DR (\%) & FPR for $90 \%$ DR (\%) & FPR for $95 \%$ DR (\%) \\
\hline \multicolumn{4}{|c|}{ SGA born at any stage } \\
\hline SGAp & $11.8(11.3-12.3)$ & $16.4(15.9-17.0)$ & $27.8(27.1-28.4)$ \\
\hline AC-M & $22.0(21.4-22.6)$ & $29.9(29.3-30.6)$ & $38.7(38.1-39.4)$ \\
\hline EFW-M & 19.3 (18.7-19.8) & $24.4(23.8-25.1)$ & 33.1 (32.5-33.8) \\
\hline EFW-M plus MF & $17.2(16.7-17.8)$ & $20.9(20.3-21.5)$ & $31.1(30.5-31.8)$ \\
\hline \multicolumn{4}{|c|}{ SGA born within 2 weeks } \\
\hline SGAp & $9.4(8.6-10.2)$ & $12.4(11.5-13.3)$ & $22.0(20.9-23.1)$ \\
\hline AC-M & $13.2(12.3-14.1)$ & $23.0(21.9-24.1)$ & 32.7 (31.5-33.9) \\
\hline EFW-M & $13.1(12.2-14.0)$ & $18.0(17.0-19.0)$ & $26.2(25.0-27.3)$ \\
\hline EFW-M plus MF & $12.6(11.7-13.5)$ & $17.0(16.0-18.0)$ & $26.1(25.0-27.3)$ \\
\hline
\end{tabular}

\section{Discussion}

\section{Main findings}

The present study confirmed that the SGAp, which was constructed using ultrasound data obtained in the second and third trimesters along with data for maternal history of hypertension and preeclampsia, showed better screening ability than EFW. The AUCs of SGAp, AC-M, EFW-M, and EFW-M plus MF for SGA neonate screening were 0.933 (95\%Cl: 0.916-0.950), 0.906 (95\% Cl: 0.887-0.925), 0.920 (95\%Cl: 0.903-0.936) and 0.925 (95\%Cl: 0.909-0.941), respectively. However, all four measures (SGAp: 0.950, 95\% Cl: 0.932-0.967; AC-M: 0.929, 95\%Cl: 0.909-0.949; EFWM: 0.938, 95\% Cl: 0.921-0.956; EFW-M plus MF: 0.941, 95\% Cl: 0.924-0.958) showed more effective screening performance if birth occurred within two weeks of the assessment.

The DRs of SGAp, AC-M, EFW-M, and EFW-M plus MF at 10\% FPR were 85.8\%, 75.8\%, 80.0\%, and 82.5\%, respectively for screening SGA neonates delivered < 2 weeks after the assessment. The FPRs of SGA screening by SGAp for $85 \%, 90 \%$, and $95 \%$ DRs were $9.4 \%, 12.4 \%$, and $22.0 \%$, respectively, in deliveries occurring $<2$ weeks after the assessment.

The DRs of SGAp, AC-M, EFW-M, and EFW-M plus MF for birth at any time were $80.4 \%, 69.6 \%, 73.8 \%$, and $74.3 \%$, respectively. The FPRs of SGA fetal screening by SGAp for $85 \%, 90 \%$, and $95 \%$ DRs were $11.8 \%, 16.4 \%$, and $27.8 \%$, respectively.

\section{Strengths and limitations of the study}

The strengths of this SGA neonatal screening study are as follows: First, based on the local median values for each GA, ultrasound data were MoM value-transformed, increasing their accuracy. Second, the study participants included pregnant women whose babies were born at 33-41 weeks (including preterm delivery). Third, the SGAp was based on prenatal ultrasound and maternal disease data, thereby ensuring better SGA screening than EFW.

The limitations of this study are as follows: First, this was a retrospective study. Of the 21,092 pregnant women who gave birth in our hospital, $3 / 4$ had been examined by ultrasound once or twice in our hospital, and most of them were tested in their local women's health care centers. Moreover, the ultrasound data were not incomplete in their local women's health care centers, resulting in a large amount of data loss. Second, the evaluations based on the SGAp model could only be performed after 33 weeks of gestation.

\section{Comparison with the findings of previous studies}

We found that EFW-M and EFW-M plus MF assessments in the third trimester could predict 73.8\% and 74.3\%, respectively, of SGA neonates delivered at 33-41 weeks' gestation at 10\% FPR. Fadigas et al. used EFW Z-score (EFW-Z) and EFW-Z plus MF data at 35-37 weeks and reported that $63.1 \%$ and $66.0 \%$ of SGA neonates ( $<10$ th percentile) were screened at $10 \%$ FPR [21]. Ciobanu et al. also used EFW-Z obtained at $35+0$ to $36+6$ weeks of gestation for screening SGA neonates (< 10th percentile) and reported DRs of $65.3 \%$ and $69.3 \%$ at $10 \%$ FPR for deliveries at $\geq 35$ weeks' gestation [22]. Bakalis et al. found that EFW-Z plus MF assessments at $30-34$ weeks could predict $79.2 \%$ and $52.7 \%$ 
of SGA neonates with $10 \%$ FPR in deliveries occurring $<5$ weeks and $>5$ weeks after the assessments, respectively [17]. Overall, the screening effect of EFW-M was similar to that of the EFW-Z. However, it is easier to convert according to the GA.

This is the first study to combine ultrasound and MF data to construct an SGAp model. The SGAp model could screen $80.4 \%$ of SGA neonates at $10 \%$ FPR in deliveries at 33-41 weeks of gestation. For deliveries that occurred within two weeks of the evaluation, the DR increased up to $85.8 \%$.

\section{Implications for clinical practice}

In China, it is common for pregnant women to undergo ultrasound examinations five times during pregnancy: at 6-8 weeks, 12-14 weeks, 23-25 weeks, 29-31 weeks, and 34-36 weeks. In addition, some pregnant women in the third trimester may undergo ultrasound examinations every month or even at two-week intervals. Appropriate use of these ultrasound data is extremely important. AC and EFW growth velocities between 20 and 36 weeks of gestation cannot be used for effective screening ${ }^{12}$. Therefore, in this study, the most effective ultrasonic data across different stages were superimposed, and a logistic regression model was used to establish the SGAp model. The screening performance of the SGAp model was shown to be better than that of EFW. The three ultrasound data points used in this study were all obtained over a relatively large gestational range, ranging from 20 to 27 weeks, 28 to 32 weeks, and 33 to 39 weeks, improving the convenience of performing SGAp-based assessments in actual clinical practice.

\section{Conclusions}

We aimed to evaluate the usefulness of the SGAp model for screening SGA neonates born at 33-41 weeks of gestation. The SGAp model could screen $80.4 \%$ of the SGA neonates at an FPR of $10 \%$. The DR increased to $85.8 \%$ if the birth time was within two weeks of the assessment. Increasing the FPR further to $16.4 \%$ improved the SGA DR to $90 \%$ at any stage. Further research is needed to determine whether a larger sample size and more refined ultrasonic data can facilitate the establishment of a more accurate SGA screening tool.

\section{Declarations}

\section{Acknowledgements}

Not applicable.

\section{Authors' contributions}

All authors worked together to design the study. Danping Xu wrote the main manuscript. Xiuzhen Shen, Heqin Guan, Minchan Yan, and Xiafang Wu collect and summarize data for statistics. Yiyang Zhu prepared figures and tables. All authors reviewed the manuscript.

\section{Funding}

This study was supported by Zhejiang Province Public Welfare Technology Application Research Project (2017C33217). The Scientific Innovation Foundation of Taizhou Hospital of Zhejiang Province (18EZB10).

\section{Availability of data and materials}

Data used or analyzed in this study are available from the corresponding author on reasonable request.

\section{Ethics approval and consent to participate}

This study was conducted in accordance with the Declaration of Helsinki and was approved by the Institutional Review Board of the Taizhou Hospital of Zhejiang Province, Wenzhou Medical University (approval number K20181112). Informed consent was obtained from all individual participants for whom identifying information was included in this article.

\section{Consent for publication}

All the authors report no disclosures relevant to the manuscript.

\section{Competing interest}

The authors declare that they have no conflict of interest.

\section{References}


1. Procas-Ramon B, Hierro-Espinosa C, Salim I et al. The impact of individual sonographer variation on the detection of small for gestational age fetuses using a third trimester growth scan. J Clin Ultrasound. 2021; 49: 442-450.

2. Ishikawa T, Uchiyama H, Baba $T$ et al. The association between congenital heart disease and small for gestational age with regard to the prevalence and outcomes. Acta Paediatr. 2021; 110: 1009-1016.

3. Langhoff-Roos J, Lindmark G. Obstetric interventions and perinatal asphyxia in growth retarded term infants. Acta Obstet Gynecol Scand Suppl. 1997; 165: 39-43.

4. Puga B, Puga PG, de Arriba A et al. Psychomotor and intellectual development (Neurocognitive Function) of children born small for gestational age (SGA). Transversal and longitudinal study. Pediatr Endocrinol Rev. 2009; 6: 358-370.

5. Lundgren EM, Tuvemo T. Effects of being born small for gestational age on long-term intellectual performance. Best Pract Res Clin Endocrinol Metab. 2008; 22: 477-488.

6. Warshafsky C, Ronzoni S, Quaglietta P et al. Comparison of sonographic fetal weight estimation formulas in patients with preterm premature rupture of membranes. BMC Pregnancy Childbirth. 2021; 21: 149.

7. Sovio U, Smith GCS. Comparison of estimated fetal weight percentiles near term for predicting extremes of birthweight percentile. Am J Obstet Gynecol. 2021; 224: 292.e1-292.e19.

8. Sánchez-Fernández M, Corral ME, Aceituno L et al. Observer Influence with Other Variables on the Accuracy of Ultrasound Estimation of Fetal Weight at Term. Medicina (Kaunas). 2021; 57: 216.

9. Patel V, Resnick K, Liang C et al. Midtrimester Ultrasound Predictors of Small-for-Gestational-Age Neonates. J Ultrasound Med. 2020; 39: 2027-2031.

10. Erkamp JS, Voerman E, Steegers EAP et al. Second and third trimester fetal ultrasound population screening for risks of preterm birth and small-size and large-size for gestational age at birth: a population-based prospective cohort study. BMC Med. $2020 ; 18: 63$.

11. Ciobanu A, Formuso C, Syngelaki A et al. Prediction of small-for-gestational-age neonates at 35-37 weeks' gestation: contribution of maternal factors and growth velocity between 20 and 36 weeks. Ultrasound Obstet Gynecol. 2019; 53: 488-495.

12. Patel V, Resnick K, Liang C et al. Midtrimester Ultrasound Predictors of Small-for-Gestational-Age Neonates. J Ultrasound Med. 2020; 39 : 2027-2031.

13. Leavitt K, Odibo L, Nwabuobi C et al. The value of introducing cerebroplacental ratio (CPR) versus umbilical artery (UA) Doppler alone for the prediction of neonatal small for gestational age (SGA) and short-term adverse outcomes. J Matern Fetal Neonatal Med. 2021; 34: 1565-1569.

14. Finneran MM, Ware CA, Russo J et al. Use of birth weight- vs. ultrasound-derived fetal weight classification methods: implications for detection of abnormal umbilical artery Doppler. J Perinat Med. 2020; 48: 615-624.

15. Birdir C, Fryze J, Frölich S et al. Impact of maternal serum levels of Visfatin, AFP, PAPP-A, sFlt-1 and PIGF at 11-13 weeks gestation on small for gestational age births. J Matern Fetal Neonatal Med. 2017; 30: 629-634.

16. Boonpiam R, Wanapirak C, Sirichotiyakul S et al. Quad test for fetal aneuploidy screening as a predictor of small-for-gestational age fetuses: a population-based study. BMC Pregnancy Childbirth. 2020; 20: 621.

17. Bakalis S, Gallo DM, Mendez 0 et al. Prediction of small-for-gestational-age neonates: screening by maternal biochemical markers at 30 34 weeks. Ultrasound Obstet Gynecol. 2015; 46: 208-215.

18. Vijayram R, Damaraju N, Xavier A et al. Comparison of first trimester dating methods for gestational age estimation and their implication on preterm birth classification in a North Indian cohort. BMC Pregnancy Childbirth. 2021; 21: 343.

19. Capital Institute of Pediatrics; Coordinating Study Group of Nine Cities on the Physical Growth and Development of Children. Growth standard curves of birth weight, length and head circumference of Chinese newborns of different gestation. Zhonghua Er Ke Za Zhi. 2020; 58: 738-746.

20. Warshafsky C, Ronzoni S, Quaglietta P et al. Comparison of sonographic fetal weight estimation formulas in patients with preterm premature rupture of membranes. BMC Pregnancy Childbirth. 2021; 21: 149.

21. Fadigas C, Saiid Y, Gonzalez R et al. Prediction of small-for-gestational-age neonates: screening by fetal biometry at 35-37 weeks. Ultrasound Obstet Gynecol. 2015; 45: 559-565.

22. Ciobanu A, Anthoulakis C, Syngelaki A et al. Prediction of small-for-gestational-age neonates at 35-37 weeks' gestation: contribution of maternal factors and growth velocity between 32 and 36 weeks. Ultrasound Obstet Gynecol. 2019; 53: 630-637.

\section{Figures}




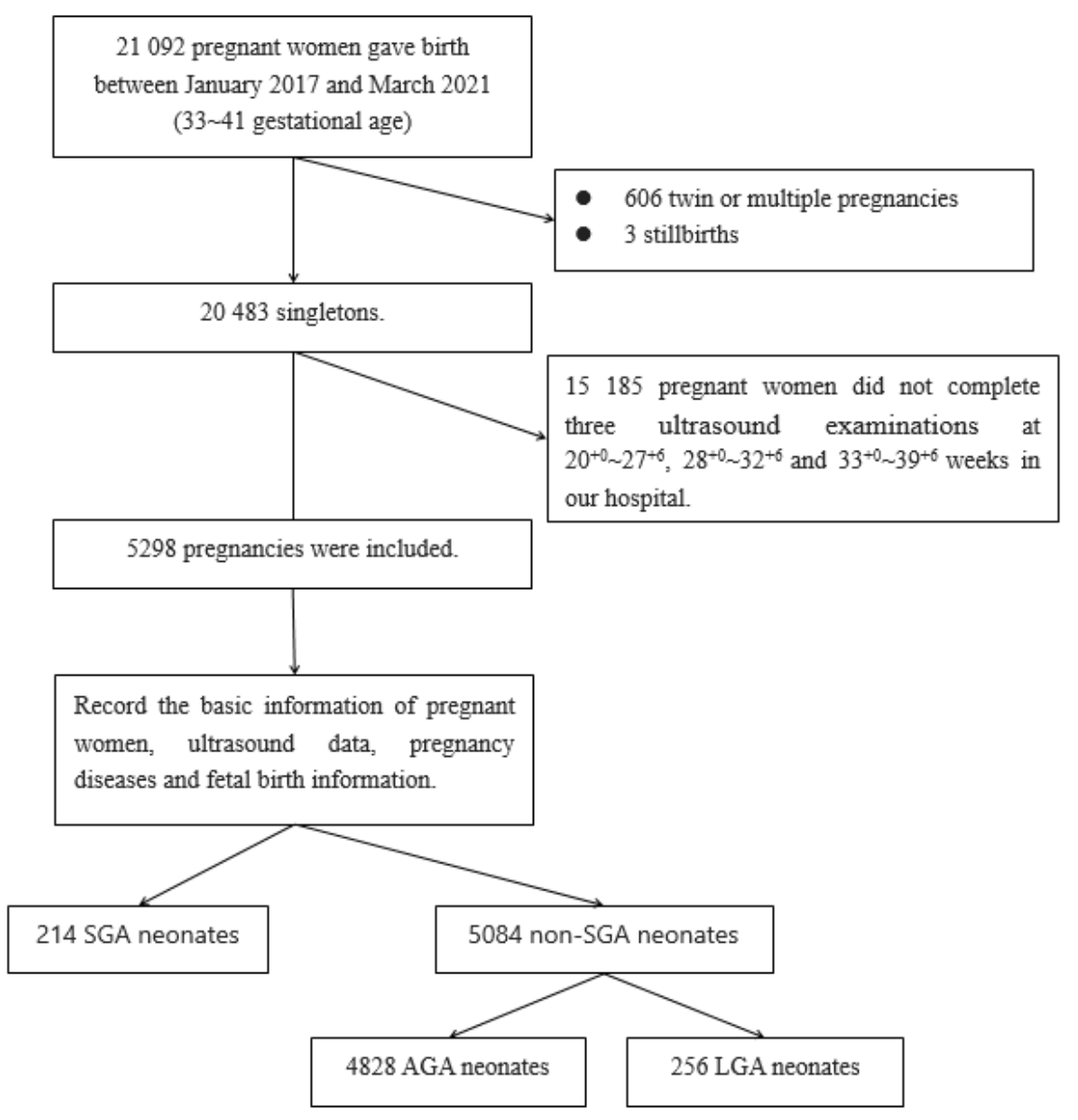

Figure 1

Flowchart of the study population. 

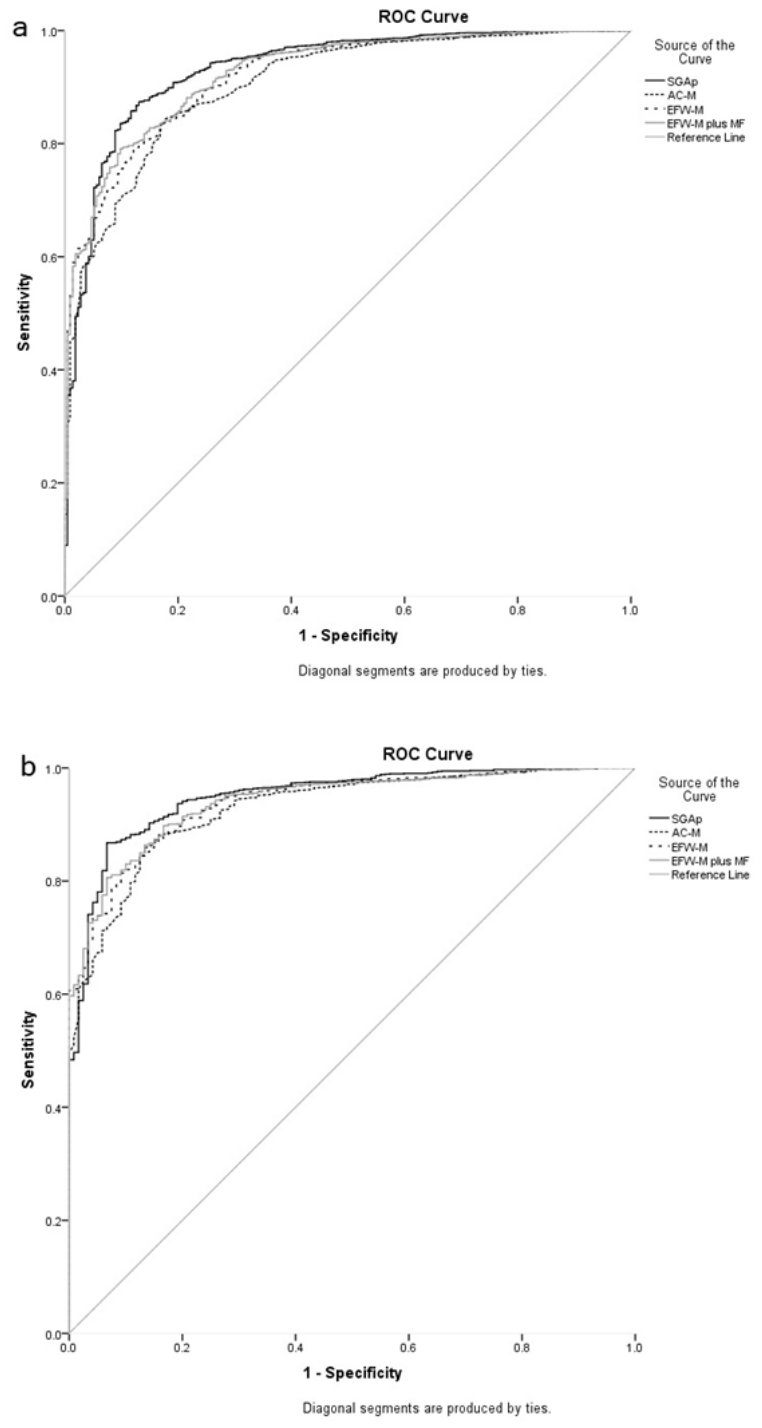

Figure 2

ROC curve analysis of SGAp, AC-M, EFW-M and EFW-M plus maternal factors in prediction of SGA neonates delivered at any time (a) or within two weeks (b) after the assessment. AC-M, AC MoM value at $33^{+0} \sim 39^{+6}$ weeks; EFW-M, EFW MoM value at $33^{+0} \sim 39^{+6}$ weeks; EFW-M plus MF, EFW-M plus maternal factors. 